\title{
Tendência e causa múltipla de óbito por insuficiência renal crônica em município da Amazônia brasileira
}

\author{
Trends and multiple causes of death due to chronic renal failure \\ in a municipality in the Brazilian Amazon
}

Thatiana Lameira Maciel Amaral ${ }^{1}$

Cledir de Araújo Amaral ${ }^{2}$

Adalberto Luiz Miranda Filho ${ }^{3}$

Gina Torres Rego Monteiro ${ }^{4}$

${ }^{1}$ Centro de Ciências da Saúde e do Desporto, Universidade Federal do Acre. Rodovia BR 364 Km 04, Distrito Industrial. 69920-900 Rio Branco AC Brasil. thatianalameira27@ gmail.com

${ }^{2}$ Instituto Federal do Acre.

Rio Branco Acre.

${ }^{3}$ Section of Cancer

Surveillance, International Agency for Research on

Cancer. Lyon France.

${ }^{4}$ Escola Nacional de Saúde Pública Sérgio Arouca, Fiocruz. Rio de Janeiro RJ Brasil.

\begin{abstract}
This study examined the mortality trend due to chronic renal failure (CRF) and verified the underlying and associated causes for this trend in the capital of the state of Acre in the Brazilian Amazon. This ecological study used data provided by DATASUS related to mortality due to CRF, which occurred between 1986 and 2012 for male and female residents of the city of Rio Branco, Acre, Brazil. The estimated annual percentage chance (EAPC) was calculated by using Poisson log-linear regression and utilizing the Joinpoint program. The results showed that the adjusted mortality rates due to CRF, with correction, ranged from 15.4 per 100,000 inhabitants in 1986 to 4.0 per 100,000 inhabitants in 2012. The EAPC was -3.5\% from 1986-2012. Deaths by CRF presented associated causes such as respiratory diseases, pneumonia and pulmonary edema, septicemias and poorly defined signs and symptoms. When CRF was analyzed as an associated cause of death, the main primary causes of death were hypertensive diseases and diabetes. Thus, there was a decrease in mortality due to CRF as an underlying cause during the period studied; however, preventive and heath care measures should be maintained.
\end{abstract}

Key words Chronic renal failure, Mortality, Cause of death
Resumo O presente estudo buscou observar a tendência de mortalidade por insuficiência renal crônica (IRC) e verificar as causas básicas e associadas na capital do Acre, Amazônia brasileira. Para tanto, foi realizado um estudo ecológico com dados de óbitos por IRC do DATASUS ocorridos entre 1986 e 2012, em ambos os sexos de residentes em Rio Branco, Acre. Estimou-se a variação anual percentual (Estimated Annual Percentage Chance-EAPC) com a técnica de regressão log-linear de Poisson do programa Joinpoint. Os resultados demonstraram que as taxas de mortalidade ajustadas da IRC com correção variaram de 15,4 por 100.000 hab., em 1986, para 4,0 por 100.000 hab., em 2012. A EAPC foi de-3,5\%, de 1986 a 2012. Os óbitos pela IRC apresentaram como causas associadas às doenças respiratórias, pneumonia e edema pulmonar, às septicemias e aos sinais $e$ sintomas mal definidos. Quando analisada a IRC como causa associada, as principais causas básicas do óbito foram as doenças hipertensivas e o diabetes. Assim, houve redução da mortalidade por insuficiência renal crônica como causa básica no período observado, contudo medidas de prevenção e assistência em saúde devem ser mantidas.

Palavras-chave Insuficiência renal crônica, Mortalidade, Causa de morte 


\section{Introdução}

A população brasileira tem vivenciado o processo de transição epidemiológica em que a mortalidade por doenças infecciosas vem declinando na última década, enquanto que os óbitos por doenças crônicas não transmissíveis crescem sendo responsáveis por $73,9 \%$ das mortes, em $2010^{1}$. As alterações no perfil das morbidades, principalmente em decorrência de mudanças desfavoráveis na dieta e atividade física da população brasileira, têm consequências importantes no padrão de mortalidade, caracterizadas pelo aumento dos óbitos em adultos e idosos por doenças não infecciosas ${ }^{2}$. Entre as principais doenças crônicas que vêm apresentando aumento na magnitude, no país, estão a hipertensão arterial e o diabetes mellitus associada a obesidade ${ }^{3}$, consideradas as principais causas da insuficiência renal crônica $(\text { IRC) })^{4-6}$.

A insuficiência renal é um grave problema de saúde pública com alta incidência e prevalência, resultando em elevados custos e alta taxa de mortalidade, podendo apresentar-se na forma aguda ou crônica ${ }^{7}$. A IRC é definida pela presença de lesão renal por período igual ou maior que três meses, caracterizada por anormalidades estruturais ou funcionais do rim, com ou sem alterações do ritmo de filtração glomerular ou por esse ser menor que $60 \mathrm{~mL} / \mathrm{min} / 1,73 / \mathrm{m}^{2}$, independentemente da lesão renal ${ }^{8}$. Suas complicações mais frequentes são as infecções, o sangramento e a insuficiência cardíaca ${ }^{9-11}$.

Além da avaliação da mortalidade por IRC como causa básica de óbito é interessante analisá-la como causa associada, visto que o processo de morte por doença crônica pode coexistir com agravos diversos, entre os quais a IRC. Dessa forma, a análise da mortalidade por causas básica e associadas permite o entendimento dos múltiplos fatores que levaram o indivíduo ao óbito, ou seja, aqueles diagnósticos que são consequências ou complicações da causa básica de morte, como as insuficiências (cardíaca, renal, hepática e outras) e a septicemia ${ }^{12}$. As doenças crônicas que são mais propensas a serem relatadas como causas associadas de morte incluem IRC, insuficiência renal não especificada, diabetes mellitus, asma, doença pulmonar obstrutiva crônica e Alzheimer. Sendo assim, quando são analisadas somente como causa básica não permitem compreender um amplo espectro de informações sobre a mortalidade ${ }^{13}$.

A análise do conjunto das causas de morte é uma forma de gerar informações sobre a etiolo- gia, evolução clínica e complicações das doenças, bem como, de forma indireta, possibilitar a formulação de ações em saúde ${ }^{14,15}$. Também, o conhecimento dos diagnósticos mencionados na declaração de óbito traz informações relevantes para o planejamento das ações de saúde ${ }^{16}$. Vale mencionar ainda o caráter inédito dos dados apresentados e a importância dessas informações corrigidas pela mortalidade por causas mal definidas e não especificadas para o conhecimento do tema. Assim, o objetivo do presente estudo foi avaliar a tendência de mortalidade por IRC e verificar as causas a ela associadas em Rio Branco, Acre.

\section{Métodos}

Trata-se de um estudo ecológico descritivo que avalia a tendência de mortalidade por IRC, no período de 1986 a 2012, e as causas múltiplas de óbitos de 1996 a 2012. Foram incluídos no estudo indivíduos de todas as idades, de ambos os sexos, residentes no município de Rio Branco, Acre, localizado na Amazônia ocidental brasileira.

As informações sobre os óbitos por IRC foram coletadas de forma desagregada do Sistema de Informação de Mortalidade (SIM), mantido pelo Ministério da Saúde (DATASUS/MS) ${ }^{17}$. Os óbitos de 1986 a 1995 foram obtidos considerando a codificação da 9a revisão da Classificação Estatística Internacional de Doenças e Problemas Relacionados à Saúde, Lesões e Causas de Óbito $(\text { CID-9 })^{18}$; para aqueles ocorridos de 1996 a 2012, tomou-se por base a $10^{a}$ revisão da Classificação Estatística Internacional de Doenças e Problemas Relacionados à Saúde (CID-10) ${ }^{19}$. Desta forma, a IRC corresponde aos códigos 585 na CID-9 e N18 na CID-10. Também foram obtidos os dados populacionais para o período em análise, no banco de dados populacionais do Instituto Brasileiro de Geografia e Estatística (IBGE), sendo as populações classificadas segundo a faixa etária ${ }^{20}$.

A proporção de causas mal definidas e sub -registros usados para mortes no Brasil continua a ser uma questão fundamental, especialmente na região Norte. Assim, para garantir a qualidade dos dados utilizados, foram obtidos dados somente da capital Rio Branco e todas as análises foram corrigidas por causas mal definidas e por insuficiência não-especificada. Para tanto, utilizou-se a metodologia de redistribuição proporcional utilizada pela Organização Mundial de Saúde (OMS) como fator de correção nos cálculos das taxas de mortalidade ${ }^{21,22}$ com redistribui- 
ção de 50\% dos óbitos cuja causa básica foi classificada como "mal definida" (códigos: 780-799 da CID-9 e R00-R99 da CID-10), critério este preconizado por estudo anterior ${ }^{12}$. Além disso, os óbitos também foram corrigidos a partir da redistribuição de todos os óbitos classificados como insuficiência renal não especificada (códigos 586 da CID-9 e N19 da CID-10), mantendo a mesma proporção dos óbitos especificados ${ }^{19}$.

Para a análise das causas do óbito foram estudadas todas as mortes nas quais a IRC foi mencionada em qualquer linha da declaração de óbito (DO). As complicações da causa básica (parte I) e as causas contribuintes (parte II) foram consideradas conjuntamente como causas associadas de morte ${ }^{23}$, cujas informações estão disponibilizadas a partir de 1996, sendo analisados os óbitos com código N18. Os diagnósticos mencionados na declaração de óbito foram agrupados conforme os capítulos do CID-10, na frequência dos diagnósticos e interesse dos pesquisadores quanto à relevância das causas, tendo sido eliminadas todas as duplicações de diagnósticos.

As causas de morte foram analisadas por meio das respectivas frequências, determinandose as causas básicas e associadas. As taxas brutas de mortalidade foram obtidas pela razão entre o número de óbitos por insuficiência renal crônica e a população estimada para cada ano da série, apresentadas por cem mil habitantes. Em seguida, essas taxas foram padronizadas por idade pelo método direto, sendo utilizada como padrão a população mundial ${ }^{24,25}$.

Posteriormente, realizou-se a análise da tendência de mortalidade por meio de modelos de regressão, utilizando a taxa global padronizada por idade e as taxas específicas por faixa etária e sexo dos óbitos por IRC. Para estruturação do modelo, as taxas padronizadas de mortalidade (y) foram consideradas como variável dependente, e os anos do período do estudo (x) como variável independente. Foi realizada regressão log-linear de Poisson, apresentando a Estimativa da Variação Anual Percentual (Estimated Annual Percentage Chance - EAPC) na qual são identificados os pontos em que há modificação da tendência. $\mathrm{O}$ modelo escolhido foi aquele com o maior número de pontos que manteve a significância estatística $(p<0,05)$. A análise de tendência também foi obtida segundo a idade e o sexo. As análises estatísticas foram realizadas no programa Joinpoint, versão 3.4 (Statistical Research and Applications Branch, National Cancer Institute, USA).

\section{Resultados}

No município de Rio Branco, entre os anos de 1986 e 2012 ocorreram 205 óbitos tendo a IRC como causa básica, destes 02 foram excluídos por faltar a informação da idade. As taxas de mortalidade por IRC, ajustadas por idade, como causa básica variaram de 10,8 por 100.000 habitantes, em 1986, para 2,6 por 100.000 habitantes, em 2012. Com as correções realizadas a partir das causas mal definidas e daquelas por insuficiência renal não especificada, as taxas de mortalidades foram de 15,4 por 100.000 habitantes, em 1986, e 4,0 por 100.000 habitantes, em 2012 (Figura 1).

A análise da variação nas taxas de mortalidade ajustadas, entre 1986 e 2012, revelou que não houve mudança de comportamento no período tendo demonstrado uma constância de decréscimo ao longo dos anos pesquisados, com EAPC da IRC de $-3,5$ (IC95\% -5,6; -1,3). A tendência decrescente manteve-se tanto na divisão por faixa etária para adultos e idosos, com EAPC de -4,6 e -2,0, respectivamente, quanto por sexo EAPC para homens de $-3,3$ e para mulheres -2,6 (Figura 1).

O número de óbitos, no período de 1996 a 2012, cuja causa básica foi insuficiência renal é de 110, tendo nesses casos em média 1,9 menções de causas por Declaração de Óbito. No entanto, quando analisada enquanto causa associada esteve presente em 319 óbitos no mesmo período. A maioria dos óbitos com registro de IRC, enquanto causa básica ou associada, ocorreu no sexo masculino na faixa etária a partir dos 60 anos (Tabela 1). Foi observado, ainda, que a mortalidade por insuficiência renal como causa básica foi maior entre as mulheres até a faixa dos 59 anos em relação aos homens (21,1\% versus $7,0 \%$ na faixa de até 39 anos e $28,9 \%$ versus $20,8 \%$, na faixa de 40 a 59 anos, entre mulheres e homens, respectivamente), entretanto, de modo inverso houve maior mortalidade em homens em todas as faixas de idade a partir dos 60 anos em comparação com as mulheres (48,6\% versus $28,9 \%$, na faixa de 60 a 79 anos e $23,6 \%$ versus $21,1 \%$, na faixa 80 anos e mais, entre homens e mulheres, respectivamente).

$\mathrm{Na}$ análise da mortalidade por IRC como causa básica, as doenças do aparelho respiratório foram as principais menções de causas, com destaque para a pneumonia $(39,7 \%)$ e edema pulmonar (17,5\%); seguidas pelos sinais e sintomas mal definidos; e pela septicemia não especificada (Tabela 2). 


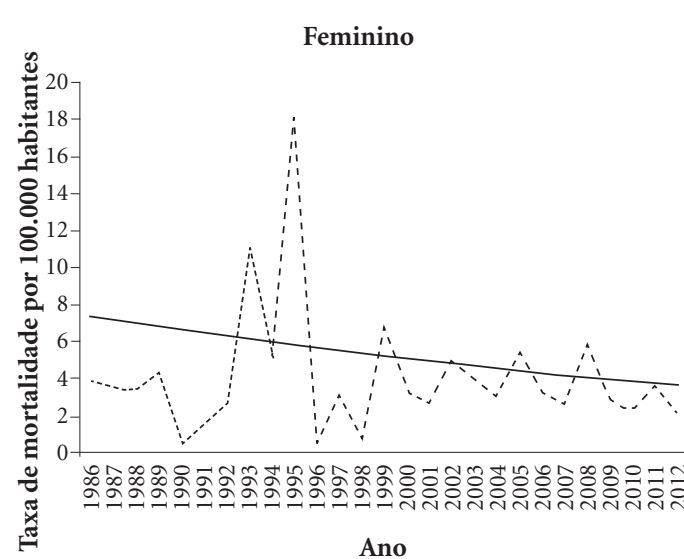

Ambos os sexos entre 50 e 59 anos

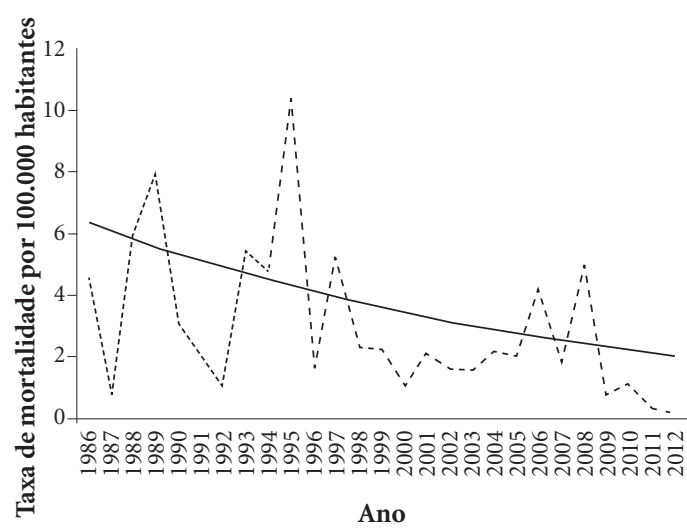

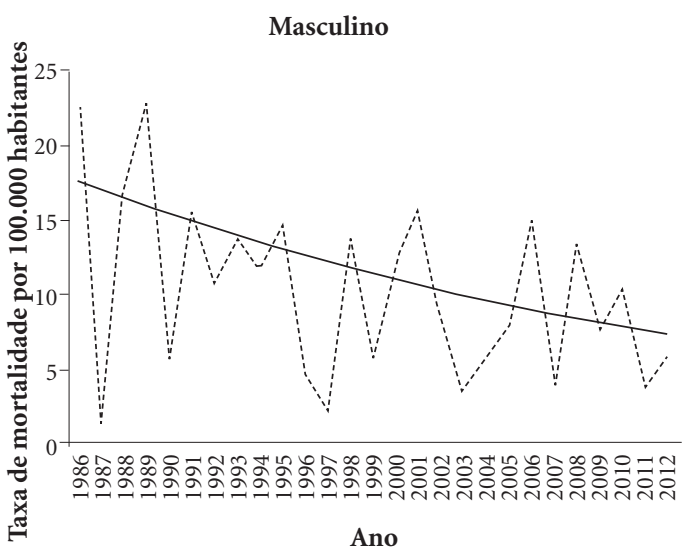

Ambos os sexos entre 50 e 59 anos

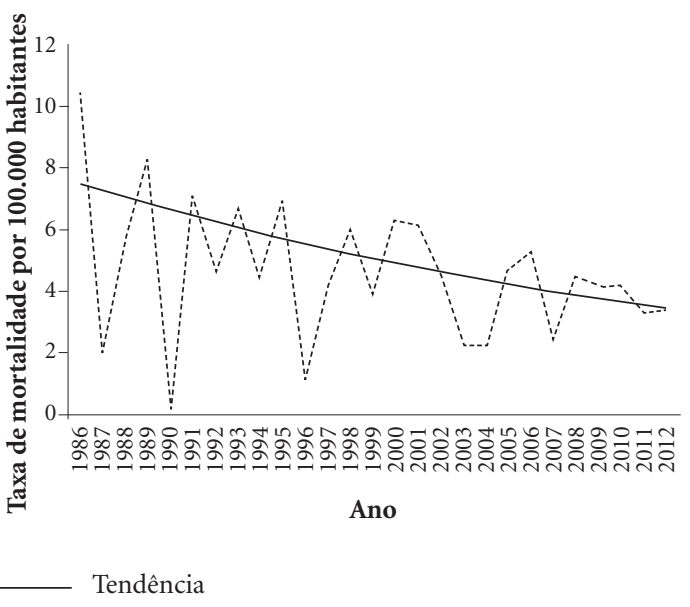

Figura 1. Tendência geral, por faixa etária e por sexo das taxas de mortalidade ajustadas com correção da insuficiência renal crônica como causa básica por 100.000 habitantes. Rio Branco-AC. 1986-2012.

Quando a IRC foi uma causa associada de óbito, as principais causas básicas foram as doenças circulatórias, seguidas das endócrinas, nutricionais e metabólicas, e das respiratórias (Tabela 3). Dentre as endócrinas, nutricionais e metabólicas destaca-se o diabetes mellitus como responsável por 22,3\% do total de óbitos.

Analisando os óbitos por doenças do aparelho circulatório, tendo a IRC como causa associada, as doenças hipertensivas representaram a principal causa do óbito seguida pelas cerebrovasculares (Tabela 4 ).

\section{Discussão}

Este trabalho observou que houve diminuição na mortalidade por IRC ao longo do período, sendo este decréscimo maior entre os idosos e as mulheres. Também verificou que entre os óbitos por insuficiência renal crônica as menções de causas foram predominantemente relacionadas ao aparelho respiratório, e quando avaliada como causa associada às doenças do aparelho circulatório foram as principais causas básicas de óbitos.

Comparativamente, as taxas de mortalidade observadas em Rio Branco foram maiores quando comparadas às taxas observadas no Brasil, no ano de 2009. Recentemente o Brasil ocupou o oitavo lugar em mortes por IRC entre os países das Américas, com taxa de mortalidade de 3,8/100.000 habitantes, em 2009, sendo de 4,8/100.000 habitantes nos homens e de $2,9 / 100.000$ habitantes nas mulheres ${ }^{26}$. A prevalência de IRC estimada no Brasil é de aproximadamente 503 pacientes por milhão da população, 
Tabela 1. Causas básica e associadas de mortalidade por insuficiência renal crônica segundo sexo e idade. Rio Branco-AC. 1996 a 2012.

\begin{tabular}{lrrrr}
\hline & Variável & \multicolumn{2}{c}{ Causa básica } & \multicolumn{2}{c}{ Causa associada } \\
\cline { 2 - 5 } & N & \% & N & $\%$ \\
\hline Sexo & & & & \\
$\quad$ Masculino & 72 & 65,5 & 214 & 67,1 \\
$\quad$ Feminino & 38 & 34,5 & 105 & 32,9 \\
Estado civil a & & & \\
$\quad$ Solteiro & 40 & 41,2 & 91 & 34,5 \\
Casado & 33 & 34,0 & 110 & 41,7 \\
Viúvo & 19 & 19,6 & 45 & 17,0 \\
Separado judicialmente & 05 & 5,2 & 18 & 6,8 \\
Idade & & & & \\
$\quad<39$ anos & 13 & 11,9 & 34 & 10,6 \\
De 40 a 59 & 26 & 23,6 & 81 & 25,4 \\
De 60 a 79 & 46 & 41,8 & 153 & 48,0 \\
De 80 a mais & 25 & 22,7 & 51 & 16,0 \\
Total & 110 & 100,0 & 319 & 100,0 \\
\hline
\end{tabular}

a causa básica $\mathrm{N}=97$; causa associada $\mathrm{N}=264$.

Fonte: Departamento de Informática do SUS (Sistema de Informações sobre Mortalidade. http://www.datasus.gov.br, acesso em 07/Mar/2014).

Tabela 2. Menções de causas referidas nos óbitos por insuficiência renal crônica como causa básica $(\mathrm{N}=110)^{\mathrm{a}}$. Rio Branco-AC. 1996 a 2012.

\begin{tabular}{lrr}
\hline \multicolumn{1}{c}{ Causas associadas de morte (CID-10) } & N & \% \\
\hline Septicemia não especificada (A41.9) & 30 & 27,3 \\
Doenças endócrinas, nutricionais e metabólicas (E00-E90) & 15 & 13,6 \\
Doenças do sistema nervoso (G00-G99) & 06 & 5,4 \\
Doenças do aparelho circulatório (I00-I99) & 24 & 21,8 \\
Doenças do aparelho respiratório (J00-J99) & 63 & 57,3 \\
Sintomas, sinais e achados anormais de exames clínicos e de laboratório, não classificados & 45 & 40,9 \\
em outra parte (R00-R99) & 28 & 25,6 \\
Outras causas associadas & 28 \\
\hline
\end{tabular}

${ }^{a}$ Porcentagens calculadas em relação ao número de óbitos por insuficiência renal.

Fonte: Departamento de Informática do SUS (Sistema de Informações sobre Mortalidade. http://www.datasus.gov.br, acessado em 07/Mar/2014).

Tabela 3. Causas básicas quando à insuficiência renal crônica é referida como causa associada na Declaração de Óbito. Rio Branco-AC. 1996 a 2012.

\begin{tabular}{|c|c|c|}
\hline Causa básica de morte (CID-10) & $\mathbf{N}$ & $\%$ \\
\hline Algumas doenças infecciosas e parasitárias (A00-B99) & 20 & 6,3 \\
\hline Neoplasias (C00-D48) & 32 & 10 \\
\hline Doenças endócrinas, nutricionais e metabólicas (E00-E90) & 88 & 27,6 \\
\hline Doenças do aparelho circulatório (I00-I99) & 113 & 35,4 \\
\hline Doenças do aparelho respiratório (J00-J99) & 29 & 9,1 \\
\hline Doenças do aparelho digestivo (K00-K93) & 15 & 4,7 \\
\hline Doenças do aparelho geniturinário (N00-N99) a & 10 & 3,1 \\
\hline Outras causas básicas & 12 & 3,8 \\
\hline Total & 319 & 100,0 \\
\hline
\end{tabular}

${ }^{a}$ Exceto diagnóstico N18 (CID-10).

Fonte: Departamento de Informática do SUS (Sistema de Informações sobre Mortalidade. http://www.datasus.gov.br, acessado em 07/Mar/2014). 
Tabela 4. Óbitos relacionados à insuficiência renal como causa associada segundo causa básica de óbitos por doenças do aparelho circulatório. Rio Branco-AC. 1996 a 2012.

\begin{tabular}{lrr}
\hline \multicolumn{1}{c}{ Doenças do aparelho circulatório (I00-I99) } & N & \% \\
\hline Doenças hipertensivas (I10-I15) & 66 & 58,4 \\
Doenças isquêmicas do coração (I20-I25) & 10 & 8,9 \\
Outras formas de doença do coração (I30-I52) & 17 & 15,0 \\
Doenças cerebrovasculares (I60-I69) & 18 & 15,9 \\
Demais doenças do aparelho circulatório & 02 & 1,8 \\
Total & 113 & 100,0 \\
\hline
\end{tabular}

Fonte: Departamento de Informática do SUS (Sistema de Informações sobre Mortalidade. http://www.datasus.gov.br, acessado em 07/Mar/2014).

e a taxa de mortalidade tendeu a cair no ano de $2012^{27}$

Nesse estudo foi observada uma tendência decrescente nas taxas de mortalidade por IRC na cidade de Rio Branco. No mundo há uma variação no comportamento da mortalidade por essa doença. Em Michigan, por exemplo, a taxa de mortalidade por IRC aumentou de 14,8/100.000 habitantes, em 1999, para 15,4/100.000 habitantes, em 2005, sendo maior entre pessoas idosas, alcançando 173,6/100.000 habitantes para aquelas com idade mais de $75 \operatorname{anos}^{28}$. No presente estudo, as maiores taxas de mortalidade também foram encontradas nos idosos.

Em um estudo de coorte realizado nos Estados Unidos (NHANES II), em 16 anos de seguimento, foi observado que indivíduos com insuficiência renal tiveram maior risco de óbito por todas as causas de morte, com predomínio das doenças cardiovasculares ${ }^{29}$. Destacam-se, também, outras causas de óbito como a septicemia, as neoplasias e a desnutrição em estudo realizado no estado do Paraná ${ }^{30}$.

Um estudo brasileiro, com dados de abrangência nacional de pacientes que iniciaram a terapia renal substitutiva em 2002 e 2003, e foram seguidos até 2004 , revelou que $42 \%$ dos pacientes em diálise peritoneal e $33 \%$ dos que estavam em hemodiálise foram a óbito, estando relacionados, principalmente, com o diabetes mellitus e as doenças cardiovasculares ${ }^{31}$. Em outro estudo sobre mortalidade por IRC, no período de 2000 a 2004, foram destacados o diabetes e as doenças cardiovasculares como as principais causas básicas de óbitos por DRC, sendo ainda observado na análise de causas associadas que $48 \%$ dos óbitos decorreram de complicações relacionadas com o tratamento, especialmente associadas a infecções ${ }^{32}$.

As principais menções de causas nos óbitos por IRC foram as doenças do aparelho respiratório, seguida por sinais e sintomas mal definidos e septicemias. A predisposição dos portadores de IRC a infecções está associada a idade, comorbidades, hipoalbuminemia, terapia imunossupressora, síndrome nefrótica, uremia, anemia e desnutrição. Muitas dessas condições podem estar presentes antes mesmo do início da diálise. Acrescido a esses fatores estão o acesso vascular e o próprio procedimento de diálise ${ }^{33}$.

As principais causas básicas de morte tendo a IRC como causa associada foram as doenças circulatórias e endócrinas, nutricionais e metabólicas, com ênfase para as doenças hipertensivas e o diabetes, corroborando outros estudos ${ }^{34-39}$. No relatório sobre mortalidade na Austrália, com dados de 2011, a IRC representou 10\% das causas múltiplas de óbitos, sendo que destas, 39\% tinham como causa associada às doenças cardiovasculares ${ }^{40}$.

A IRC promove a progressão da doença cardiovascular por meio da expansão de volume, distúrbios do metabolismo do cálcio-fosfato, dislipidemia e hipertensão arterial. Por outro lado, a doença cardiovascular pode promover o declínio da função renal por meio da insuficiência cardíaca e da aterosclerose ${ }^{41}$. Nesse contexto, os estudos sobre causas múltiplas da mortalidade favorecem o entendimento sobre a IRC com vistas a prevenção dos fatores relacionados ao óbito ${ }^{13}$. Vale ressaltar que os dados apresentados no presente estudo referem-se a indivíduos portadores de insuficiência renal crônica e que, portanto, apresentam maior risco de morte.

Vale mencionar como limitação do estudo a utilização de um banco de dados secundário, sujeito a imprecisões. Para minimizar essa questão, fez-se o cálculo das taxas com a correção por causas mal definidas e por insuficiência renal não especificada. Outra possível limitação é a qualidade das informações que depende do preenchimento completo e correto da declaração de óbito ${ }^{42}$. Entretanto, como pontos positivos do estudo 
destacam-se a análise das causas de morte por IRC, enquanto causa básica e associada, e a possibilidade de delineamentos de ações no intuito de minimizar as complicações a elas relacionadas que resultam em morte na cidade de Rio Branco, contribuindo para o acúmulo de conhecimentos que favoreçam a adoção de medidas de maior abrangência adequadas às realidades locais.

A utilização não somente da causa básica, mas também das causas associadas, possibilita a identificação das doenças que participam do processo mórbido do óbito por IRC. Assim, compreender a evolução temporal e as causas envolvidas no processo de morte possibilita dimensionar o problema de saúde pública que é a insuficiência renal crônica.

Assim conclui-se que houve redução na mortalidade por IRC em Rio Branco, sendo a diminuição mais acentuada entre os maiores de 50 anos de idade e entre as mulheres no período. Os óbitos causados pela IRC apresentaram como menções de causas as doenças respiratórias, dentre elas a pneumonia e o edema pulmonar, bem como os achados anormais e as septicemias. E quando analisada como causa associada, as causas básicas dos óbitos mais frequentes foram as doenças hipertensivas e o diabetes.

\section{Colaboradores}

TLM Amaral, CA Amaral e AL Miranda Filho contribuiram na concepção do projeto de pesquisa, coleta, processamento, análise, interpretação dos dados e redação do manuscrito; GTR Monteiro, participou da análise e interpretação dos dados, revisão crítica e aprovação da versão final do manuscrito.

\section{Referências}

1. Duarte EC, Barreto SM. Transição demográfica e epidemiológica: a Epidemiologia e Serviços de Saúde revisita e atualiza o tema. Epidemiol Serv Saúde [Internet]; 2012 [cited 2016 Out 23]; 21(4):529-532. Available from: http://scielo.iec.pa.gov.br/scielo.php?script=sci_arttext\&pid $=$ S167949742012000400001\&lng $=$ pt http://dx.doi.org/10.5123/S1679-49742012000400001

2. Schmidt MI, Duncan BB, Silva GA, Menezes AM, Monteiro CA, Barreto SM, Chor D, Menezes PR. Chronic non-communicable diseases in Brazil: burden and current challenges. Lancet 2011; 377(9781):1949-1961.

3. Schmidt MI, Duncan BB, Hoffmann JF, Moura L, Malta DC, Carvalho RMSV. Prevalência de diabetes e hipertensão no Brasil baseada em inquérito de morbidade auto-referida, Brasil, 2006. Rev Saude Publica 2009; 43(Supl. 2):74-82.

4. Balbi AL, Gabriel DP, Barsante RC, Caramori JT, Martin LC, Barreti P. Mortalidade e prognóstico específico em pacientes com insuficiência renal aguda. Rev Assoc Medica Bras 2005; 51(6):318-322.

5. Bortolotto LA. Hipertensão arterial e insuficiência renal crônica. Rev Bras Hipertens 2008; 15(3):152-155.

6. Tsioufis C, Tatsis I, Thomopoulos C, Wilcox C, Palm F, Kordalis A, Katsiki N, Papademetriou V, Stefanadis C. Effects of hypertension, diabetes mellitus, obesity and other factors on kidney haemodynamics. Curr Vasc Pharmacol 2014; 12(3):537-548.

7. Levey AS, Eckardt KU, Tsukamoto Y, Levin A, Coresh J, Rossert J, De Zeeuw D, Hostetter TH, Lameire N, Eknoyan G. Definition and classification of chronic kidney disease: a position statement from Kidney Disease: Improving Global Outcomes (KDIGO). Kidney Int 2005; 67(6):2089-2100.
8. National Kidney Foundation. K/DOQI clinical practice guidelines for chronic kidney disease: evaluation, classification, and stratification. Am J Kidney Dis 2002; 39(2 Supl. 1):S1-266.

9. Costa JAC, Vieira-Neto OM, Moysés Neto M. Insuficiência renal aguda. Medicina 2003; 36(2/4):307-324.

10. Naqvi SB, Collins AJ. Infectious complications in chronic kidney disease. Adv Chronic Kidney Dis 2006; 13(3):199-204.

11. Thomas R, Kanso A, Sedor JR. Chronic kidney disease and its complications. Prim Care 2008; 35(2):329-vii.

12. Jorge MHPM, Gotlieb SLD, Laurenti R. The national mortality information system: problems and proposals for solving them I - Deaths by natural causes. Rev Bras Epidemiol 2002; 5(2):197-211.

13. Australian Institute of Health and Welfare (AIHW). Multiple causes of death in Australia: an analysis of all natural and selected chronic disease causes of death 19972007 [Internet]. Canberra: AIHW; 2012 [cited 2014 May 16]. (AIHW bulletin no. 105. Cat. no. AUS 159). Available from: http://www.aihw.gov.au/WorkArea/ DownloadAsset.aspx?id $=10737422598$

14. Israel RA, Rosenberg HM, Curtin LR. Analytical potential for multiple cause-of-death data. Am J Epidemiol 1986; 124(2):161-179.

15. Redelings MD, Sorvillo F, Simon P. A comparison of underlying cause and multiple causes of death: US vital statistics, 2000-2001. Epidemiology 2006; 17(1):100103.

16. Ishitani LH, França E. Doenças crônico-degenerativas em adultos da região centro-sul de Belo Horizonte: análise sob a perspectiva de causas múltiplas de morte. Inf Epidemiol Sus 2001; 10(4):177-188. 
17. Brasil. Ministério da Saúde (MS). Sistema de Informação de mortalidade/DATASUS [Internet]. 2012 [cited 2014 Aug 16]. Available from: http://www2.datasus. gov.br/DATASUS/index.php?area $=0205$

18. Organização Mundial da Saúde (OMS). Classificação estatística internacional de doenças: manual de lesões e causas de óbito - CID-9. 9a ed. São Paulo: Centro Brasileiro Classificação Doenças em Português; 1979. (Manual de instrução).

19. Organização Mundial da Saúde (OMS). Classificação estatística internacional de doenças e problemas relacionados à saúde - CID -10 [Internet]. 10a ed. São Paulo: Centro Colaborador da OMS para a Classificação de Doenças em Português/Edusp; 1993. (Manual de instrução; vol. 2). Available from: http://www.datasus.gov. $\mathrm{br} / \mathrm{cid} 10 / \mathrm{V} 2008 / \mathrm{cid} 10 . \mathrm{htm}$

20. Instituto Brasileiro de Geografia e Estatística (IBGE). Sistema IBGE de Recuperação Automática - SIDRA [Internet]. 2013 [cited 2014 May 4]. Available from: http:// www.sidra.ibge.gov.br/cd/cd2010RgaAdAgsn.asp

21. França E, Teixeira R, Ishitani L, Duncan BB, Cortez-Escalante JJ, Morais OL, Szwarcwald CL. Ill-defined causes of death in Brazil: a redistribution method based on the investigation of such causes. Rev Saude Publica 2014; 48(4):671-681.

22. Mathers CD, Bernard C, Iburg KM, Inoue M, Fat DM, Shibuya K, Stein C, Tomijima N, Xu H. Global burden of disease in 2002: data sources, methods and results. Geneva: World Health Organization; 2003.

23. Santo AH. Causas múltiplas de morte: formas de apresentação e métodos de análise [Tese]. São Paulo: Universidade de São Paulo, Faculdade de Saúde Pública; 1988.

24. Doll R, Payne P, Waterhouse JAH. Cancer incidence in five continents. Geneva: Union Internationale Contre le Cancer; 1966. Vol. I

25. Segi M. Cancer mortality for selected sites in 24 countries (1950-57). Sendai: Department of Public Health, Tohoku University of Medicine Japan; 1960.

26. Pan American Health Organization (PAHO). Visualizing renal failure and chronic kidney diseases age-standardized mortality rate in countries of the Americas, 2000-2009 [Internet]. Washington: PAHO; 2014. [cited 2015 May 16]. (Non-communicable Diseases and Mental Health). Available from: http://www.paho. org/hq/index.php?option=com_content\&view $=$ article\&id=9381:renal-failure-chronic-kidney-disease-ck$\mathrm{d} \&$ Itemid $=41166$ \&lang $=$ en

27. Sesso RC, Lopes AA, Thome FS, Lugon JR, Watanabe Y, Santos DR. Report of the Brazilian Chronic Dialysis Census 2012. J Bras Nefrol 2014; 36(1):48-53.

28. Centers for Disease Control and Prevention. Kidney disease mortality - Michigan, 1989-2005. MMWR Morb Mortal Wkly Rep 2007; 56(10):225-227.

29. Muntner P, He J, Hamm L, Loria C, Whelton PK. Renal insufficiency and subsequent death resulting from cardiovascular disease in the United States. J Am Soc Nephrol 2002; 13(3):745-753.

30. Peres LA, Biela R, Herrmann M, Matsuo T, Ann HK, Camargo MT, Rohde NR, Uscocovich VS. Epidemiological study of end-stage kidney disease in western Paraná: an experience of 878 cases in 25 years. J Bras Nefrol 2010; 32(1):51-56.
31. Szuster DAC, Caiaffa WT, Andrade EIG, Acurcio FA, Cherchiglia ML. Survival analysis of dialysis patients in the Brazilian Unified National Health System. Cad Saude Publica 2012; 28(3):415-424.

32. Siviero PCL, Machado CJ, Cherchiglia ML. Chronic kidney failure by means of multiple causes of death in Brazil. Cad Saude Colet 2014; 22(1):75-85.

33. Dalrymple LS, Go AS. Epidemiology of acute infections among patients with chronic kidney disease. Clin J Am Soc Nephrol 2008; 3(5):1487-1493.

34. Global Burden of Metabolic Risk Factors for Chronic Diseases Collaboration. Cardiovascular disease, chronic kidney disease, and diabetes mortality burden of cardiometabolic risk factors from 1980 to 2010: a comparative risk assessment. Lancet Diabetes Endocrinol 2014; 2(8):634-647.

35. Perazella MA, Khan S. Increased mortality in chronic kidney disease: a call to action. Am J Med Sci 2006; 331(3):150-153.

36. Tonelli M, Wiebe N, Culleton B, House A, Rabbat C, Fok M, McAlister F, Garg AX. Chronic kidney disease and mortality risk: a systematic review. J Am Soc Nephrol 2006; 17(7):2034-2047.

37. Chronic Kidney Disease Prognosis Consortium, Matsushita K, van der Velde M, Astor BC, Woodward M, Levey AS, de Jong PE, Coresh J, Gansevoort RT. Association of estimated glomerular filtration rate and albuminuria with all-cause and cardiovascular mortality in general population cohorts: a collaborative meta-analysis. Lancet 2010; 375(9731):2073-2081.

38. Rao MV, Qiu Y, Wang C, Bakris G. Hypertension and CKD: Kidney Early Evaluation Program (KEEP) and National Health and Nutrition Examination Survey (NHANES), 1999-2004. Am J Kidney Dis 2008; 51(4 Supl. 2):S30-S37.

39. Rashidi A, Sehgal AR, Rahman M, O'Connor AS. The case for chronic kidney disease, diabetes mellitus, and myocardial infarction being equivalent risk factors for cardiovascular mortality in patients older than 65 years. Am J Cardiol 2008; 102(12):1668-1673.

40. Australian Institute of Health and Welfare (AIHW). Cardiovascular disease, diabetes and chronic kidney disease: Australian facts: mortality [Internet]. Canberra: AIHW; 2014 [cited 2014 Oct 11]. (Cardiovascular, diabetes and chronic kidney disease series no. 1. Cat. no. CDK 1). Available from: http://www.aihw.gov.au/ WorkArea/DownloadAsset.aspx?id=60129549107

41. Weiner DE, Sarnak MJ. Chronic kidney disease and cardiovascular disease: A bi-directional relationship? Dial Transplant 2007; 36(3):113-120.

42. Conselho Federal de Medicina. Resolução CFM n ${ }^{\circ}$ 1.779 , de 11 de novembro de 2005. Regulamenta a responsabilidade médica no fornecimento da Declaração de Óbito. Diário Oficial da União 2005; 5 dez.

Artigo apresentado em 23/08/2016

Aprovado em 08/11/2016

Versão final apresentada em 10/11/2016 